\title{
INVOLUTIONS ON A TROPICAL LINE
}

\author{
JOAQUIM ROÉ*
}

\begin{abstract}
Tropical involutive linear maps and pencils of degree 2 on the tropical projective line are introduced and studied. Both concepts are related in a similar way to linear involutions and $g_{2}^{1}$ 's in the classical (algebraic) projective line, and tropicalization relates the algebraic phenomena to the tropical ones.
\end{abstract}

\section{Introduction}

\subsection{Complete linear series}

Complete linear series on tropical curves have been recently object of intense study, and are relatively well understood thanks to Riemann-Roch type theorems and to combinatoric descriptions, see [5], [1], [2]. These linear series turn out to be polyhedral complexes which do not have pure dimension, and this fact has been an obstacle to define and study non-complete linear series. As far as we know, [6] and [3], which study linear series of plane curves (determined by imposing a singular point in the first case, three base points in the second) are the only works dealing with non-complete linear series so far. On projective tropical space, complete linear series are specially simple, as they consist of a single polytope, a simple quotient of a free tropical module. Thus it seems reasonable to consider sublinear series of these (as in [6] and [3]), and the simplest case is that of pencils of degree 2 on the projective line. In classical algebraic geometry, these are called $g_{2}^{1}$ 's on $\mathrm{P}^{1}$, and they are very closely related to linear involutions, i.e., projectivities of $\mathrm{P}^{1}$ onto itself whose square is the identity, and to (ramified) double covers of $\mathrm{P}^{1}$ by itself. In this note we introduce tropical notions of $g_{2}^{1}$ and involutive linear maps, and show that both are closely related as in the algebraic case. Our description suggests that a double cover of the tropical line by itself could have a segment of "ramification points"; in the forthcoming work [7] we study involutions on the Berkovich projective line and show that its (ramified) double covers by itself have a whole (tropical) line of ramification points.

\footnotetext{
* Partially supported by the Spanish Ministerio de Ciencia e Innovacion project MTM200910359.

Received 16 April 2010, in final form 21 November 2011.
} 


\subsection{Setting}

Let $\mathrm{R}_{\text {trop }}=\mathrm{R} \cup\{-\infty\}$ be the tropical semifield, with operations $a \oplus b=$ $\max (a, b)$ and $a \odot b=a+b$. Natural number exponents in tropical expressions should be interpreted tropically, i.e., $a^{n}=a \odot \cdots \odot a$. A tropical polynomial in the variables $x_{1}, \ldots, x_{n}$ is a formal expression $\bigoplus_{\alpha} a_{\alpha} \odot x^{\alpha}$ where $\alpha \in Z_{\geq 0}^{n}$ is a multi-index, $x^{\alpha}=x_{1}^{\alpha_{1}} \odot \cdots \odot x_{n}^{\alpha_{n}}$ and $a_{\alpha} \in \mathbf{R}_{\text {trop }}$ for all $\alpha$. Such a tropical polynomial defines, by interpreting sums and products tropically, a piecewise linear function with integer slopes

$$
\left(x_{1}, \ldots, x_{n}\right) \mapsto \bigoplus_{\alpha} a_{\alpha} \odot x^{\alpha}=\max _{\alpha}\left(a_{\alpha}+\sum_{i=1}^{n} \alpha_{i} x_{i}\right),
$$

whose corner locus, denoted $\mathscr{T}\left(\bigoplus_{\alpha} a_{\alpha} x^{\alpha}\right)$, is a tropical affine hypersurface.

Tropical projective $n$-space, $\operatorname{TP}^{n}$, is the quotient of $\mathrm{R}_{\text {trop }}^{n+1} \backslash\{(-\infty, \ldots,-\infty)\}$ by the equivalence relation $\left(x_{1}, \ldots, x_{n}\right) \sim\left(y_{1}, \ldots, y_{n}\right)$ when $\exists t \in \mathrm{R}, x_{i}=$ $y_{i}+t$ for all $i$.

We use the notation $\left(x_{0}: \ldots: x_{n}\right)$ for the point of $\mathrm{TP}^{n}$ corresponding to $\left(x_{0}, \ldots, x_{n}\right) \in \mathrm{R}_{\text {trop }}^{n}$. A tropical polynomial $P$ does not define a function on $\mathrm{TP}^{n}$, but if it is homogeneous then its corner locus is well defined, and we also denote it $\mathscr{T}(P)$. A pair of homogeneous tropical polynomials of the same degree, not both equal to $-\infty$, define a rational function $\left(x_{0}: \ldots: x_{n}\right) \mapsto$ $\left(P\left(x_{0}, \ldots, x_{n}\right): Q\left(x_{0}, \ldots, x_{n}\right)\right)$ (a continuous piecewise linear map to the projective line). Since a polynomial different from $-\infty$ can take the value $\infty$ only at the borders of projective space, i.e., when at least one of the $x_{i}=-\infty$, rational function can have a nonempty indeterminacy locus (where $(P: Q)$ takes the non-existent value $(-\infty:-\infty)$ ) but it must be a subset of the border; in the case $n=1$ such indeterminacies can always be resolved, extending the function by continuity.

As a set, $\mathrm{TP}^{1}$ can be identified with $\mathrm{R} \cup\{-\infty,+\infty\}$ by mapping $(a: b)$ to $b-a$. We use the notation $p_{-}=(0:-\infty)$ and $p_{+}=(-\infty: 0)$ for the two ends, or points at infinity, of TP ${ }^{1}$. Note that $\mathrm{TP}^{1}$ is a totally ordered set, the order being $(a: b) \leq(c: d)$ when $b-a \leq d-c$. Given two points $p, q \in \operatorname{TP}^{1}$ we denote by $[p, q]$ the tropical convex hull [4] of the pair, which is just the segment with ends at $p$ and $q$.

\subsection{A divisor}

A divisor on $\mathrm{TP}^{1}$ is an element $\sum_{p \in \mathrm{TP}^{1}} a_{p} \cdot p$ of the free abelian group on its points, and it is effective when all coefficients $a_{p}$ are non-negative. The degree of a divisor is the sum of the coefficients. The order of a rational function $f=(P: Q)$ at a point $p \in \operatorname{TP}^{1}$ is defined, following [5], as the sum of the 
outgoing slopes of $f$ at $p$, and

$$
(f)=\sum_{p \in \mathrm{TP}^{1}} \operatorname{ord}_{p}(f) \cdot p
$$

is the principal divisor determined by $(f)$. Two divisors are linearly equivalent if they differ by a principal divisor.

Given a divisor $D$, one considers the space $R(D)$ of all rational functions $f$ such that $(f)+D$ is effective, and the complete linear series $|D|=\{(f)+D \mid$ $f \in R(D)\}$. Actually, this machinery makes sense mostly for curves of higher genus, because in the case of a line $|D|$ is just the set of all effective divisors of the same degree, but it is worth keeping the $\mathrm{R}_{\text {trop-module and geometric }}$ structure of $R(D)$ and $|D|$ also in this case.

Thus, for any divisor $D$ of degree $2,|D|=\left|2 \cdot p_{+}\right|$, and a rational function such that $(f)+2 \cdot p_{+}$is effective can always be written as $f(x: y)=\left(x^{2}\right.$ : $P(x, y))$, where $P(x, y)=\alpha_{20} \odot x^{2} \oplus \alpha_{11} \odot x \odot y \oplus \alpha_{02} \odot y^{2}$ is a homogeneous tropical polynomial of degree 2 . So the complete linear series of degree 2 is

$$
|D|=\left\{\begin{array}{l|l}
\mathscr{T}\left(\alpha_{20} \odot x^{2} \oplus \alpha_{11} \odot x \odot y \oplus \alpha_{02} \odot y^{2}\right) & \begin{array}{l}
\alpha_{20}, \alpha_{11}, \alpha_{02} \in \mathbf{R}_{\text {trop }} \\
\alpha_{20} \oplus \alpha_{11} \oplus \alpha_{02} \neq-\infty
\end{array}
\end{array}\right\} .
$$

Observe that this is not isomorphic to the tropical projective plane but to a quotient of it, because two tropical polynomials can have the same roots even if one is not a scalar multiple of the other; indeed, given $\alpha_{20}, \alpha_{02}$, for all $\alpha_{11} \leq\left(\alpha_{20}+\alpha_{02}\right) / 2, \mathscr{T}\left(\alpha_{20} \odot x^{2} \oplus \alpha_{11} \odot x \odot y \oplus \alpha_{02} \odot y^{2}\right)=\left(\alpha_{02} / 2: \alpha_{20} / 2\right)$.

\section{Tropical involutions}

\subsection{Tropical matrices and projectivities}

In the set $\mathrm{TM}_{2}$ of all $2 \times 2$ matrices with entries in $\mathrm{R}_{\text {trop }}$, not all equal to $-\infty$, consider the equivalence relation

$$
A \sim B \text { when } \exists t \in \mathrm{R}, A=B+\left(\begin{array}{ll}
t & t \\
t & t
\end{array}\right) .
$$

Let $\mathrm{PTM}_{2}$ be the set of matrices in $\mathrm{TM}_{2}$ with no column equal to $\left(\begin{array}{l}-\infty \\ -\infty\end{array}\right)$ modulo $\sim$. Given $A \in \mathrm{TM}_{2}$ with no column equal to $\left(\begin{array}{l}-\infty \\ -\infty\end{array}\right)$, there is an associated map $\varphi_{A}: \mathrm{TP}^{1} \rightarrow \mathrm{TP}^{1}$ given by multiplication on the left, and $\varphi_{A}=\varphi_{B} \Leftrightarrow A \sim B$.

We define the sign of a matrix as

$$
\sigma\left(\begin{array}{ll}
a_{00} & a_{10} \\
a_{01} & a_{11}
\end{array}\right)=\operatorname{sign}\left(a_{00}+a_{11}-a_{10}-a_{01}\right) \in\{-1,0,1\} .
$$


2.2. MAIN PROPERTIES OF THE SIGN Let $A, B \in \mathrm{TM}_{2}$ with no column equal to $\left(\begin{array}{c}-\infty \\ -\infty\end{array}\right)$.

(1) If $A \sim B$ then $\sigma(A)=\sigma(B)$, i.e., the sign of a matrix in $\mathrm{PTM}_{2}$ is well-defined.

(2) $\sigma(A \odot B) \in\{0, \sigma(A) \sigma(B)\}$.

(3) $\sigma(A)=0 \Leftrightarrow \varphi_{A}$ is constant.

(4) $\sigma(A)=1 \Leftrightarrow \varphi_{A}$ is order-preserving, i.e., $p \geq q \Rightarrow \varphi_{A}(p) \geq \varphi_{A}(q)$.

(5) $\sigma(A)=-1 \Leftrightarrow \varphi_{A}$ is order-reversing, i.e., $p \geq q \Rightarrow \varphi_{A}(p) \leq \varphi_{A}(q)$.

The proof of 2.2 is elementary.

\subsection{Involutive matrices}

In PTM 2 , consider the subset ITM ${ }_{2}$ of those matrices with 2 equal values in the diagonal, i.e., of the form $J_{a, b, c}=\left(\begin{array}{ll}b & a \\ c & b\end{array}\right)$. These form the tropicalization of the locus of involutive matrices in PGL(2), and we call them involutive tropical matrices. Denote $\varphi_{a, b, c}=\varphi_{J_{a, b, c}}$.

2.4. Characterization Let $A \in \mathrm{PTM}_{2}$, and let $F=\operatorname{Im}\left(\varphi_{A}\right) \subset \mathrm{TP}^{1} . A$ is involutive if and only if $F$ is not reduced to an end of $\mathrm{TP}^{1}$ and the restriction of $\varphi_{A}^{2}$ to $F$ is the identity map.

Proof. By Develin-Sturmfels [4], the image of $\varphi_{A}$ is the tropical convex hull of the two points determined by the columns of $A$, i.e., a point or a segment of positive length. Thus, $\varphi_{a, b, c}$ is surjective (bijective in fact) if and only if $b=-\infty$ or $a=c=-\infty$. Now for all $(x: y) \in \operatorname{TP}^{1}, \varphi_{-\infty, b,-\infty}(x: y)=$ $(b \odot x: b \odot y)=(x: y)$, i.e., $\varphi_{-\infty, b,-\infty}$ is the identity map. On the other hand, $\varphi_{a,-\infty, c}^{2}(x: y)=\varphi_{a,-\infty, c}(a \odot y: c \odot x)=(a \odot c \odot x: a \odot c \odot y)=(x: y)$, so $\varphi_{a,-\infty, c}^{2}$ is the identity map, for all $a, c \in \mathrm{R}$.

It is not hard to check that $\varphi_{a, b, c}$, restricted to its image, is always a bijection, but we distinguish three different cases, depending on the sign of the matrix.

$\sigma\left(J_{a, b, c}\right)=1$ : This includes all cases when $a$ or $c$ is $-\infty$, i.e., when the image contains an end of TP ${ }^{1}$. In this case, a computation as above shows that $\varphi_{a, b, c}$ restricted to $[(b: c),(a: b)]$ is the identity, whereas $[(0:-\infty),(b: c)]$ maps identically to $(b: c)$ and $[(a: b),(-\infty: 0)]$ maps to $(a: b)$. Moreover $\varphi_{a, b, c}$ is idempotent, i.e., $\varphi_{a, b, c}^{2}=\varphi_{a, b, c}$. Topologically speaking $\varphi_{a, b, c}$ is the retraction of $\mathrm{TP}^{1}$ to $F$.

$\sigma\left(J_{a, b, c}\right)=0$ : In this case $\varphi_{a, b, c}$ is constant and the image point is not an end of TP ${ }^{1}$. Therefore, trivially, it is idempotent and restricted to its image it is the identity map. 
$\sigma\left(J_{a, b, c}\right)=-1$ : This includes all cases with $b=-\infty$; these are involutions and have been considered before, so assume $b \in \mathbf{R}$. In this case $a, c \in \mathrm{R}$, and one checks that $\varphi_{a, b, c}$ restricted to $[(a: b),(b: c)]$ is the unique orientation-reversing isometry, whereas $[(0:-\infty),(a: b)]$ maps identically to $(b: c)$ and $[(b: c),(-\infty: 0)]$ maps to $(a: b)$. Moreover $\varphi_{a, b, c}^{2}=\varphi_{-a,-b,-c}$ is the retraction of $\operatorname{TP}^{1}$ to $F$.

Conversely, let $A=\left(\begin{array}{ll}a_{00} & a_{10} \\ a_{01} & a_{11}\end{array}\right)$ be a matrix such that the restriction of $\varphi_{A}^{2}$ to $F=\operatorname{Im}\left(\varphi_{A}\right)$ is the identity map. Write $A^{2}=\left(\begin{array}{ll}b_{00} & b_{10} \\ b_{01} & b_{11}\end{array}\right)$. If

$$
A^{2}\left(\begin{array}{l}
x \\
y
\end{array}\right)=\left(\begin{array}{llll}
b_{00} \odot x & \oplus b_{10} \odot y \\
b_{01} \odot x & \oplus b_{11} \odot y
\end{array}\right)=\left(\begin{array}{l}
x \\
y
\end{array}\right)
$$

in a segment of positive length, we must have $b_{00} \odot x \oplus b_{10} \odot y=b_{00} \odot x$ (i.e., $y-x<b_{00}-b_{10}$ ) and $b_{01} \odot x \oplus b_{11} \odot y=b_{11} \odot y$ (i.e., $y-x>b_{01}-b_{11}$ ) in that segment, and $b_{00}=b_{11}$. So we can assume without loss of generality that $b_{00}=b_{11}=0$ and $b_{10}+b_{01}<0$, i.e., $A^{2}=J_{b_{10}, 0, b_{01}}$. The equation

$$
A^{2}=\left(\begin{array}{cc}
a_{00}^{2} \oplus a_{10} \odot a_{01} & a_{10} \odot\left(a_{00} \oplus a_{11}\right) \\
a_{01} \odot\left(a_{00} \oplus a_{11}\right) & a_{10} \odot a_{01} \oplus a_{11}^{2}
\end{array}\right)=\left(\begin{array}{cc}
0 & b_{10} \\
b_{01} & 0
\end{array}\right)
$$

has two types of solutions, depending on whether $0=2 a_{00}>a_{10}+a_{01}$ or $2 a_{00} \leq a_{10}+a_{01}=0$. If $0=2 a_{00}>a_{10}+a_{01}$ then one is forced to have $2 a_{11}=0$ and so $A=A^{2}=J_{a_{10}, 0, a_{01}}$ is one of the retractions above, $\sigma(A)=1$.

All other solutions have $a_{10}+a_{01}=0$ and $a_{00}, a_{11} \leq 0$. The condition that $\varphi_{A}^{2}$ is the identity on $F$ implies that each column of $A$ determines a point in the segment $\left[\left(0: b_{01}\right),\left(b_{10}: 0\right)\right]$ where $\varphi_{A}^{2}$ is the identity, therefore

$$
\begin{aligned}
a_{01}+\max \left(a_{00}, a_{11}\right) & =\quad a_{01} \odot\left(a_{00} \oplus a_{11}\right)=b_{01}-0 \leq a_{11}-a_{10}, \\
-a_{10}-\max \left(a_{00}, a_{11}\right) & =-\left(a_{10} \odot\left(a_{00} \oplus a_{11}\right)\right)=0-b_{10} \leq a_{01}-a_{00},
\end{aligned}
$$

so $\max \left(a_{00}, a_{11}\right) \leq a_{11}-a_{01}-a_{10}=a_{11}$ and $\max \left(a_{00}, a_{11}\right) \leq a_{00}-a_{01}-a_{10}=$ $a_{00}$. Thus $a_{00}=a_{11} \leq a_{10}+a_{01}$, which shows $A$ is involutive, and either constant or of negative sign.

\section{Tropical pencils}

3.1. $A g_{2}^{1}$ on $\mathrm{TP}^{1}$

A $g_{2}^{1}$ on $\mathrm{TP}^{1}$ must be a linear subseries of the complete linear series of degree 2 which, as explained in 1.3 , is

$$
\left|2 \cdot p_{+}\right|=\left\{\mathscr{T}\left(\alpha_{20} \odot x^{2} \oplus \alpha_{11} \odot x \odot y \oplus \alpha_{02} \odot y^{2}\right) \mid \alpha_{20}, \alpha_{11}, \alpha_{02} \in \mathbf{R}_{\text {trop }}\right\},
$$


Since a tropical line in a plane is given by a tropical linear equation, we define $g_{2}^{1}$ 's as determined by a tropical linear equation in the coefficients $\alpha_{20}, \alpha_{11}, \alpha_{02} \in \mathbf{R}_{\text {trop }}$. Thus, if $L_{a, b, c}$ denotes the tropical line defined as the corner locus of the tropical polynomial function $a \odot \alpha_{20} \oplus b \odot \alpha_{11} \oplus c \odot \alpha_{02}$, the corresponding $g_{2}^{1}$ is

$$
\begin{aligned}
g_{a, b, c} & =\left\{\mathscr{T}\left(\alpha_{20} \odot x^{2} \oplus \alpha_{11} \odot x \odot y \oplus \alpha_{02} \odot y^{2}\right) \mid\left(\alpha_{20}: \alpha_{11}: \alpha_{02}\right) \in L_{a, b, c}\right\} \\
& \subset\left|2 \cdot p_{+}\right| .
\end{aligned}
$$

3.2. CorresPondenCE PENCILS $\leftrightarrow$ INVOLUTIONS Let $a, b, c \in \mathbf{R}_{\text {trop }}$, with $a \oplus b \neq-\infty \neq b \oplus c$. Then the $\operatorname{set}\left\{p+\varphi_{a, b, c}(p)\right\}_{p \in \mathrm{TP}^{1}} \subset\left|2 p_{+}\right|$is equal to $g_{a, b, c}$.

Proof. The proof is slightly different depending on the sign of the involutive matrix $J_{a, b, c}$. Let us prove the statement for $\sigma\left(J_{a, b, c}\right) \geq 0$, and leave the negative case to the reader. Let $F$ be the image of $\varphi_{a, b, c}$; its end points are $(b: c)$ and $(a: b)$, and its mid point is $(a / 2: c / 2)$.

We first prove that $\left\{p+\varphi_{a, b, c}(p)\right\}_{p \in \mathrm{TP}^{1}} \subset g_{a, b, c}$. Distinguish four cases, depending on the position of the point $p \in \operatorname{TP}^{1}$ relative to these three points.

$p \leq(b: c)$ : Put $p=(b: t)$ with $t \leq c$, then $p+\varphi_{a, b, c}(p)=(b: t)+(b:$ $c)=\mathscr{T}\left(c \odot t \odot x^{2} \oplus b \odot c \odot x \odot y \oplus b^{2} \odot y^{2}\right)$, and $\left(c \odot t: b \odot c: b^{2}\right) \in L_{a, b, c}$ because $a \odot c \odot t \leq b \odot b \odot c=c \odot b^{2}$.

$(b: c) \leq p \leq(a / 2: c / 2)$ : Put $p=(b: t)$ with $c \leq t \leq b+(c-a) / 2$, then $p+\varphi_{a, b, c}(p)=2(b: t)=\mathscr{T}\left(t^{2} \odot x^{2} \oplus b \odot c \odot x \odot y \oplus b^{2} \odot y^{2}\right)$, and $\left(t^{2}: b \odot c: b^{2}\right) \in L_{a, b, c}$ because $a \odot t^{2} \leq b \odot b \odot c=c \odot b^{2}$.

$(a / 2: c / 2) \leq p \leq(a: b)$ : Put $p=(t: b)$ with $a \leq t \leq b+(a-c) / 2$, then $p+\varphi_{a, b, c}(p)=2(t: b)=\mathscr{T}\left(b^{2} \odot x^{2} \oplus a \odot b \odot x \odot y \oplus t^{2} \odot y^{2}\right)$, and $\left(b^{2}: a \odot b: t^{2}\right) \in L_{a, b, c}$ because $a \odot b^{2}=b \odot a \odot b \geq c \odot t^{2}$.

$p \geq(a: b)$ : Put $p=(t: b)$ with $t \leq a$, then $p+\varphi_{a, b, c}(p)=\{(a: b),(t:$ $b)\}=\mathscr{T}\left(b^{2} \odot x^{2} \oplus a \odot b \odot x \odot y \oplus a \odot t \odot y^{2}\right)$, and $\left(b^{2}: a \odot b:\right.$ $a \odot t) \in L_{a, b, c}$ because $a \odot b^{2}=b \odot a \odot b \geq c \odot a \odot t$.

Along the way we have shown that equations parameterized by two of the three rays in $L_{a, b, c}$ (namely, $a+\alpha_{20} \leq b+\alpha_{11}=c+\alpha_{02}$ and $a+\alpha_{20}=$ $\left.b+\alpha_{11} \geq c+\alpha_{02}\right)$ do give pairs of points $p+\varphi_{a, b, c}(p)$. The third ray, with equations $a+\alpha_{20}=c+\alpha_{02} \geq b+\alpha_{11}$ corresponds to the polynomials $c \odot x^{2} \oplus t \odot x \odot y \oplus a \odot y^{2}$ with $t \leq(a+c) / 2$, whose unique double root is the mid point $(a / 2: c / 2) \in F$, fixed by $\varphi_{a, b, c}$, so we are done. 


\section{Tropicalization}

\subsection{Tropicalization of involutions}

Next we check that all tropical involutive maps (and $g_{2}^{1}$ 's) as defined above arise as tropicalizations of algebraic involutions. Let $K$ be a valued field, with value group $G \subset \mathrm{R}$, and denote the valuation by $v$. Assume furthermore that $v(2)=0$. Given $a, b, c \in G \cup\{-\infty\}$, with $a \oplus b \neq-\infty \neq b \oplus c$, choose $\alpha, \beta, \gamma \in K$ such that $v(\alpha)=-a, v(\beta)=-b, v(\gamma)=-c$. Since there are fields with value grup equal to R, the restriction to $G$ is not actually relevant; rather, it is considered here only for completeness.

Consider the matrix $A=\left(\begin{array}{cc}\beta & \alpha \\ \gamma & -\beta\end{array}\right)$; if it turns out to be singular (which can indeed happen if $a+c=2 b$ ) replace $\alpha$ by some $\alpha+x$ with $x \neq 0$, $v(x)>a$, which certainly exist. Since $A^{2}=\left(\begin{array}{cc}\beta^{2}+\alpha \gamma & 0 \\ 0 & \beta^{2}+\alpha \gamma\end{array}\right), A$ is the matrix of an involution in $\mathrm{P}_{K}^{2}$, and its tropicalization is the involutive tropical matrix $\left(\begin{array}{ll}b & a \\ c & b\end{array}\right)$.

It is clear that the $g_{2}^{1}$ on $\mathrm{P}_{K}^{1}$ corresponding to the involution with matrix $A$ tropicalizes to the tropical $g_{2}^{1}$ corresponding to the tropicalized matrix. It is more illustrative to pay some attention to the fixed points of the involution.

4.2. Tropicalization OF FIXED POINTS Let $\bar{K}$ be an algebraic closure of $K$, and let $p, q \in \mathrm{P}_{\bar{K}}^{1}$ be the fixed points of the involution $\varphi_{A}: \mathrm{P}_{\bar{K}}^{1}$ determined by $A=\left(\begin{array}{cc}\beta & \alpha \\ \gamma & -\beta\end{array}\right)$. Let $A^{t}=\left(\begin{array}{ll}b & a \\ c & b\end{array}\right)=-v(A)$ be the tropicalization of $A$. Then $\sigma\left(A^{t}\right)=1$ if and only if $v(p) \neq v(q)$, and in this case the ends of the segment fixed by the tropical involutive map are $-v(p)$ and $-v(q)$.

Proof. $p=(x: y)$ and $q=\left(x^{\prime}: y^{\prime}\right)$ are the zeros of the homogeneous polynomial $\gamma x^{2}-2 \beta x y-\alpha y^{2}$, so their tropicalizations $-v(p),-v(q)$ are the tropical roots of the tropicalization $F=c \odot x^{2} \oplus b \odot x \odot y \oplus a \odot y^{2}$. These are distinct if and only if $2 b \geq c+a$, i.e., if and only if $\sigma\left(A^{t}\right)=1$. In such a case, the columns of $A^{t}$ are exactly the tropical roots of $\mathrm{F}$, and they are also the ends of the segment fixed by the tropical involutive map.

\section{REFERENCES}

1. Baker, M., and Norine, S., Riemann-Roch and Abel-Jacobi theory on a finite graph, Adv. Math. 215 (2007), 766-788.

2. Baker, M., and Norine, S., Harmonic morphisms and hyperelliptic graphs, Int. Math. Res. Not. 15 (2009), 2914-2955.

3. Brodsky, S. B., and Sturmfels, B., Tropical quadrics through three points, Linear Algebra Appl. 435 (2011), 1778-1785. 
4. Develin, M., and Sturmfels, B., Tropical convexity, Doc. Math. 9 (2004), 1-27 (electronic), correction: ibid., pp. 205-206.

5. Gathmann, A., and Kerber, M., A Riemann-Roch theorem in tropical geometry, Math. Z. 259 (2008), 217-230.

6. Markwig, H., Markwig, T., and Shustin, E., Tropical curves with a singularity in a fixed point, Manuscripta Math. 137 (2012), 383-418.

7. Martínez, C., and Roé, J., Involutions on berkovich and tropical lines, in preparation.

UNIVERSITAT AUTÒNOMA DE BARCELONA

DEPARTAMENT DE MATEMÀTIQUES

E-08193 BELLATERRA (BARCELONA)

SPAIN

E-mail: jroe@mat.uab.cat 